\title{
Análisis comparativo del costo del kWh de plantas de potencia utilizando como combustible biomasa de los pinos afectados por el Dendroctonus Frontalis en Honduras
}

Fernando José Zorto Aguilera ${ }^{1}$

Wilson Alonso Licona Martínez ${ }^{2}$

\section{RESUMEN}

En Honduras en el 2106 se presentó un problema de aprovechamiento de madera de pino afectada por el Dendroctunus Frontalis, el cual afectó a 389.024.38 ha de pino. Por tal motivo es necesario una solución viable a dicho problema, por lo cual se propone el estudio de ubicación de dos escenarios para el manejo de la biomasa. En el Escenario 1 se desea instalar una planta con capacidad de 50MW y para el Escenario 2 se instala zonificado dos plantas de potencia una de $35 \mathrm{MW}$ y otra de $25 \mathrm{MW}$ para verificar, cuál de las dos soluciones genera un costo unitario de Kilowatio-hora más bajo. En este caso, se evaluaron cuatro tipos de tecnologías de plantas de potencia para recudir al máximo el flujo de combustible en cada escenario. En este caso el Escenario 2 nos presenta los mejores valores de costo unitarios de kilowatio-hora siendo estos en la planta de potencia CR3REG2REC para la número 1 con capacidad de $35 \mathrm{MW}$ netos y en la planta de potencia CR3REG2REC número 2 con capacidad de $25 \mathrm{MW}$ netos en US\$ 0.0543/kWh y 0.0799 US\$/kWH respectivamente.

Palabras clave: dendroctunus frontalis, biomasa, costos, plantas de potencias, Honduras

\footnotetext{
${ }^{1}$ Profesor Auxiliar del Departamento de Ingeniería Mecánica, Facultad de Ingeniería, Universidad Nacional Autónoma de Honduras

${ }^{2}$ Ingeniero Electricista Facultad de Ingeniería, Universidad Nacional Autónoma de Honduras
} 


\section{ABSTRACT}

In 2015 , at Honduras there was a problem of using pine wood affected by dendroctunus frontalis, (spread over a $389,024.38$ ha of pine). For this reason, the location study of the scenarios for the management of the biomass was proposed, in order to present a viable solution to the problem. In scenario 1, a plant with a capacity of 50 MW would be installed, and for the scenario 2, a system with two power plants of 35 MW and another of $25 \mathrm{MW}$ would be installed to verify which of the two solutions generated a lower unitary cost per kilowatt/hour. In this case, four types of power plant technologies were evaluated to indicate the best fuel flow in each scenario. Therefore, scenario 2 presented the best unit cost values per kilowatt/ hour, being $35 \mathrm{MW}$ net in CR3REG2REC power plant number 1 (US \$ $0.0543 / \mathrm{kWh}$ ) and $25 \mathrm{MW}$ net in CR3REG2REC power plant number 2 (US \$ 0.0799 / kWH).

Keywords: dendroctunus frontalis, bio-mass, costs, power plants, Honduras 


\section{INTRODUCCIÓN}

En el año 2016, los bosques cubrían un área aproximada de 5,384,424.88 ha en el territorio de Honduras. Según la evaluación nacional forestal (ENF) se tiene una cantidad de 5,046985.27 de bosque Latifoliado y Conífera; el bosque mixto y el mangle cubre una extensión del $6.3 \%$ del bosque con 337,439.61 ha (ICF, 2017). Dentro de este reporte de ICF no se consideró el área afectada por el Dentroctunus Frontalis (D. Frontalis). Según el CONADEH (2016), la plaga de D. Frontalis desde el mes de abril del 2015, siguió un avance de 20 ha/día, en dirección de este-oeste, desde el municipio de Gualaco hasta los demás municipios afectados por el brote. Según datos del ICF (2015) existió una superficie afectada de 389.024.38 ha, siendo una cantidad de 33,969,607.15 m3 de bosque afectado por la plaga, este recurso natural tiene es una fuente interesante de energía para ser utilizada. Esto se puede ver en la figura 1.

Figura 1. Mapa general de áreas activas e inactivas del Ataque de Plaga, diciembre del 2015

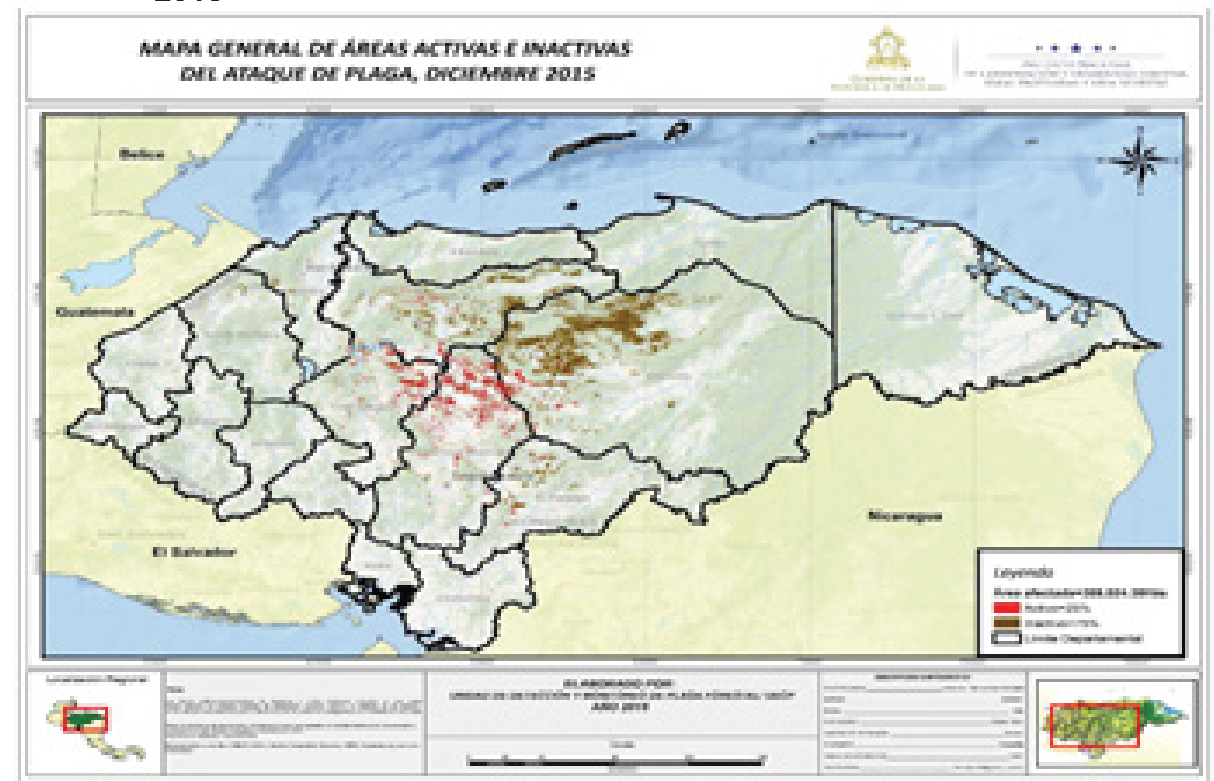

Fuente: ICF, Anuario Estadístico 2015.

EI D. Frontalis es uno de los insectos que mayores daños ha ocasionado en el sur de

Estados Unidos de Norteamérica, donde las pérdidas oscilan en el orden de $\$ 900$ millones en daños en pinos desde 1960 hasta 1990 (Prices, T.S., 1990). La actividad 
de este insecto agresivo es atacar al árbol alojándose entre la corteza y la madera. En esta zona el gorgojo va dejando surcos o gallerías serpenteantes. Así mismo, Dicho insecto es el vector de un hongo azul, el cual coloniza la xilema, bloqueando el flujo de agua dentro del árbol, esto ocasiona la muerte del árbol (Thatcher \& Connor, 1985). En el Año 2015, La República de Honduras por medio del Decreto Ejecutivo Número PCM-051-2015, declara en el país una "Emergencia Forestal a Nivel Nacional por el Ataque de la Plaga del Gorgojo de Pino". En el artículo cinco de dicho decreto, instruye al ICF a promover del uso de la biomasa forestal para la generación de energía. Las múltiples especies de pino en el Honduras son consideradas una fuente de energía por su alto contenido energético. El poder calorífico superior del pino se encuentra definido en 17,098 kj/kg según los cálculos obtenidos por Parikh, Channiwala, y Ghosal (2005) en su correlación matemática. La alternativa para la producción de Energía Eléctrica por medio de la Biomasa se pueden utilizar ciclos termodinámicos tipo Rankine (Rafael Barreda del Campo, Augusto Araújo da Gama Cerqueira, \& Nebra, 1998; Taner \& Sivrioglu, 2017); las cuales funcionan como un ciclo de potencia que consume combustible sólido, líquido o gaseoso para transferir calor al agua y generar una cambio de fase liquido vapor, donde este funciona como fluido de trabajo para mover una turbina y generar potencia(Çengel, 2012)..

Sin embargo, existen otros ciclos de potencia que implementa calderas que queman biomasa o queman ambos combustibles para obtener el calor emitido por la combustión de la madera para luego ser transferida a un fluido de trabajo para logar mover una turbina y posteriormente producir energía eléctrica(Saidur, Abdelaziz, Demirbas, Hossain, \& Mekhilef, 2011). Esto conlleva analizar parámetros termodinámicos y económicos para determinar cuál de estos es el más viable para implementarse(Kaushik, Reddy, \& Tyagi, 2011). Por tal motivo, el objetivo del estudio es realizar una comparación entre los costos de kwh entre varias plantas de potencias de ciclo termodinámico tipo Rankine, las cuales utilicen como combustible la biomasa de los pinos afectados por el D. Frontalís. Para lograr dicho análisis se debe implementar la metodología de la termoeconómia, la cual genera un estudio de los procesos irreversibles de la planta de potencia e identifica los costos de producción totales de la generación de energía (Uysal et al., 2017)

\section{METODOLOGÍA}

Para definir el análisis comparativo del costo de Kwh de plantas de potencia para la madera de pino con la plaga del $\mathrm{D}$. Frontalis, se utilizó la información del anuario 
estadístico forestal editado por el ICF (2015) para obtener la cantidad de hectáreas de bosque de pino plagadas en los departamentos de Olancho, Yoro, Francisco Morazán, Comayagua y El Paraíso, por ser los departamentos con mayor cantidad de biomasa disponibles para el uso energético según el decreto ejecutivo PCM-051-2015. Según ICF (2015), la tasa de corta anual promedio es de 49.8950 $\mathrm{m} 3 / \mathrm{ha}$, con pinos con una densidad promedio de 0.5181 ton $/ \mathrm{m} 3$. Esto sirvió para cuantificar los metros cúbicos de biomasa a utilizando, por medio de la siguiente ecuación:

$B A=A^{*} \beta$

Donde BA es la Biomasa afectada en $\mathrm{m} 3$, A es el área de pino afectada en ha y $ß$ es la tasa de corta permisible por hectárea

Obtenida la cantidad volumen de biomasa es necesario conocer dónde se ubicará la planta de generación de energía eléctrica. Para tal motivo, se seleccionó por departamento un centro de distribución para enviar la madera a la planta de potencia, los cuales se encuentran en la Ciudad de Comayagua, Talanga, Danli, Olanchito y Gualaco. Esto se observa en la figura 2.

Figura 2. Centros de distribución de madera afectada para su envió a la planta de potencia.

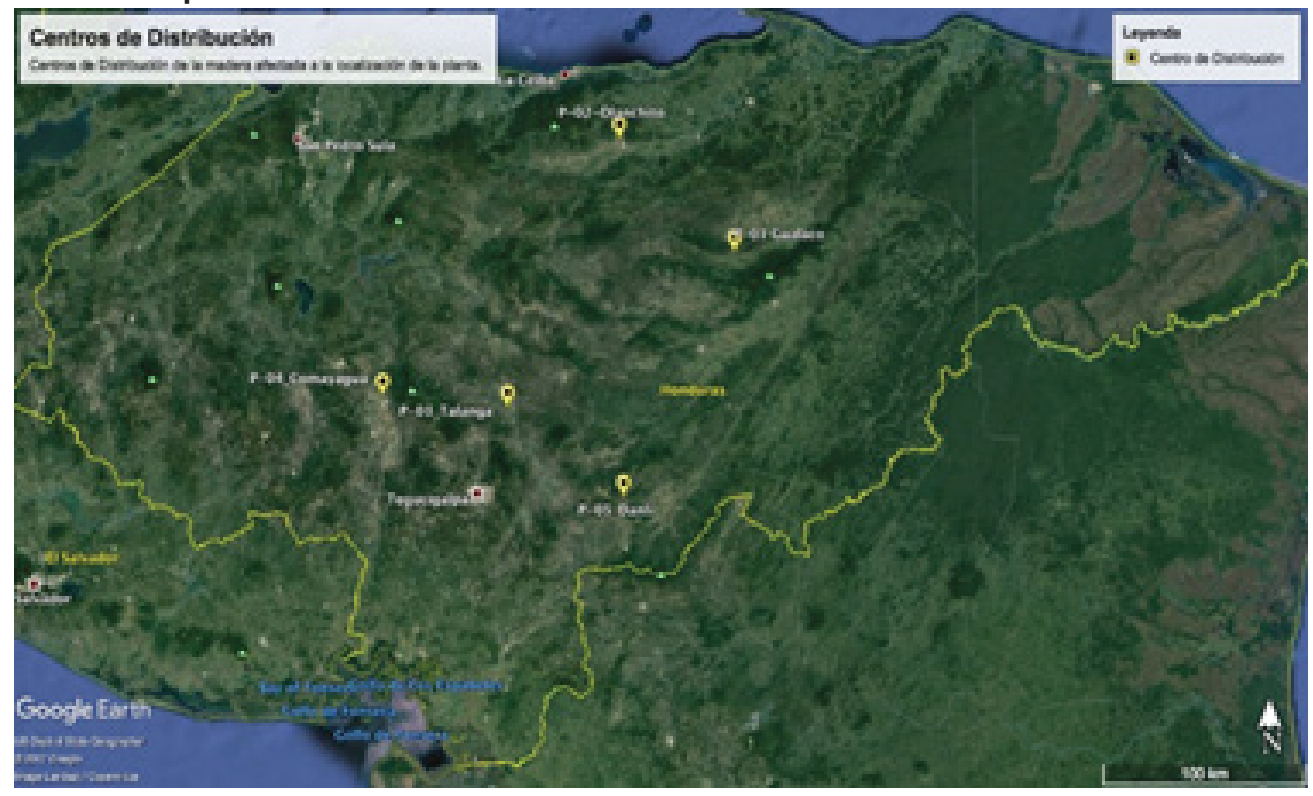

Fuente: Elaboración propia 
Para obtener la mejor ubicación de la planta utilizamos el método del centro de gravedad, el cual se basa en el cálculo de transporte para cada uno de los centros de distribución que servirán para enviar la madera a la fábrica. Con este método garantizamos el costo más bajo para el transporte de dicha madera(Oxford Dictionary, 2018). En este caso, el precio negociado para transporte es el mismo para cada punto, el cual es de $\$ 8 /$ ton. Es de suma importancia aclarar que utilizamos el mismo criterio del centro de gravedad, pero ligado al volumen de masa que se transportará a la fábrica y no al precio, puesto que es el mismo para cada localización. Esto nos garantiza tener más cerca la planta del punto con mayor volumen de biomasa. Para dicho cálculo utilizamos la ecuación 2:

$$
\left(\overline{\mathrm{X}}_{i}, \overline{\mathrm{Y}}_{i}\right)=\left(\frac{\sum_{\mathrm{xi}{ }^{*} \mathrm{v} i}}{\sum_{\mathrm{v} i}}, \frac{\sum_{\mathrm{y}^{*} v i}}{\sum_{\mathrm{v} i}}\right)
$$

Donde $\mathrm{xi}, \mathrm{yi}, \mathrm{X}_{i}, \mathrm{Y}_{i}$ y vi son las coordenadas en latitud y longitud de cada centro de distribución i, las coordenadas en latitud y longitud de la Planta de Potencia y el volumen de biomasa a transportar desde cada centro de distribución i a la planta respectivamente (Ballou, 1973).

El costo de transporte al sitio es directamente proporcional a la distancia recorrida para el cálculo de dicho valor. Por tal razón, se determina mediante una aproximación de la carretera asumiendo son muy serpenteantes. Con base en lo anterior, el valor de la distancia será tomado por la siguiente ecuación:

$$
\mathrm{CT}=\mathrm{F}^{*} \mathrm{D}
$$

Donde la CT, F y D son costo de transporte en US\$ $/ \mathrm{m3}$, precio de transporte en US\$ por $\mathrm{Km}^{*} \mathrm{~m} 3$ siendo US\$ $0.3335 / \mathrm{km}^{*} \mathrm{~m} 3$ y la distancia línea del centro de distribución a la planta en $\mathrm{km}$ respectivamente. En la Figura 3. Se muestra más detallado el esquema de la aproximación de la distancia.

Figura 3. Modelo matemático para la aproximación de la distancia de las carreteras en Honduras.

Daprox $=1.5 \mathrm{D}$

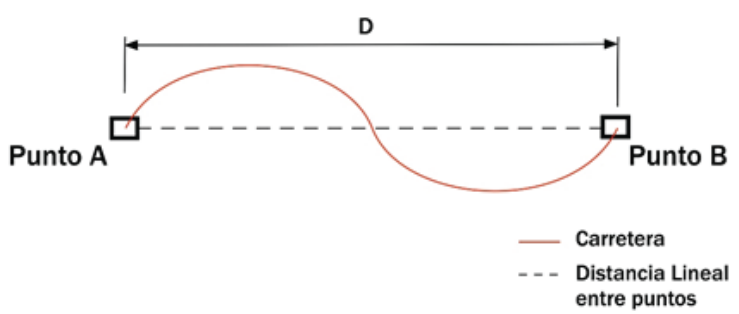

Fuente: elaboración propia 
Conocido el costo de transporte por m3 es necesario determinar el costo promedio total de la madera por $\mathrm{kg}$ puesto en planta, por lo que utilizamos la siguiente ecuación:

$$
C T_{\text {prom }}=\sum_{i=1}^{n} \frac{C T_{i}+C u m_{i}}{n \rho_{i}}
$$

Donde $C T_{\text {prom }}, C T_{i}, C u m_{i}, \rho_{i}$ y $n$ son el costo promedio total de la madera por $\mathrm{kg}$, el Costo de transporte por centro de distribución en US\$/m3, el costo del m3 de madera por centro de distribución de $\$ 8.50 / \mathrm{m3}$, la densidad de la especie de madera en $\mathrm{kg} / \mathrm{m} 3$ y el número de centros de distribución respectivamente (Whalley, Klein, \& Benjamin, 2017).

Posteriormente al resultado obtenido por el precio unitario del combustible en US\$/kg se tomó la decisión de realizar dos escenarios: a) Focalizando la distribución de la madera a una única planta de generación; y b) Realizando la ubicación para dos plantas por diferente clúster de distribución de madera. El primer clúster de distribución será Comayagua, Talanga y Danli; y el segundo por Gualaco y Olanchito.

Dentro de dichas localizaciones se analizaron cuatro plantas de generación de energía eléctrica para evaluar cuál de ellas brindaría mejores resultados de costos de kWh en US\$ para su operación. Dentro de las plantas propuestas fueron: a) Ciclo Rankine simple (CS); b) Ciclo Rankine con Regeneración de Contacto Directo (CRRD); c) Ciclo Rankine con Recalentamiento y Regeneración de Contacto de Superficie como de Contacto Directo (CR2REGREC); y d) Ciclo Rankine con dos Recalentamiento, dos regeneraciones de contacto de superficie y de Contacto Directo (CR3REG2REC). Así como se observa en la figura 4. No obstante, es importante aclarar que las extracciones son calculadas por medio de la temperatura óptima de extracción. Los datos utilizados para las diferentes plantas fueron los utilizados en la tabla 1.

Como parámetro de comparación entre las diferentes opciones de Escenarios y plantas de potencias será el costo unitario de kilowatt hora para determinar cuál es la mejor opción entre ellos. La fórmula que se utilizó fue:

$$
C U_{k W h}=3600 \frac{i v+C T_{\text {prom }}}{\rho W_{\text {neta }}}
$$

Donde $v$ caudal de biomasa utilizado en $\mathrm{m}^{3}$, CTprom costo promedio total de la madera en US\$ $/ \mathrm{kg}, \rho$ la densidad de la madera en $\mathrm{kg} / \mathrm{m} 3$ y $w_{\text {neta }}$ potencia neta total del Ciclo Termodinámico. 
Tabla 1. Datos del análisis energético en sus escenarios 1 y 2

\begin{tabular}{|l|l|l|l|}
\hline \multirow{2}{*}{$\begin{array}{l}\text { Datos de las diferentes } \\
\text { plantas }\end{array}$} & Escenario 1 & \multicolumn{2}{|l|}{ Escenario 2 } \\
\cline { 2 - 4 } & Planta Única & Planta 1 & Planta 2 \\
\hline Potencia neta: & $5000 \mathrm{~kW}$ & $35000 \mathrm{~kW}$ & $25000 \mathrm{~kW}$ \\
Eficiencia de la Turbina $\left(\eta_{\mathrm{t}}\right.$ & 0.9 & 0.9 & 0.9 \\
Eficiencia de la bomba $\left(\eta_{\mathrm{b}}\right)$ & 0.8 & 0.8 & 0.8 \\
Eficiencia de la Caldera: & 0.8 & 0.8 & 0.8 \\
Densidad del Pino: & $3.81 \mathrm{~kg} / \mathrm{m}^{3}$ & $3.81 \mathrm{~kg} / \mathrm{m}^{3}$ & $3.81 \mathrm{~kg} / \mathrm{m}^{3}$ \\
Presión de Alta: & $15000 \mathrm{kPa}$ & $15000 \mathrm{kPa}$ & $15000 \mathrm{kPa}$ \\
Presión de baja: & $100 \mathrm{kPa}$ & $100 \mathrm{kPa}$ & $100 \mathrm{kPa}$ \\
Temp. de recalentado: & $550{ }^{\circ} \mathrm{C}$ & $550{ }^{\circ} \mathrm{C}$ & $550{ }^{\circ} \mathrm{C}$ \\
Temp. de recalentamiento: & $550{ }^{\circ} \mathrm{C}$ & $550{ }^{\circ} \mathrm{C}$ & $550{ }^{\circ} \mathrm{C}$ \\
\hline
\end{tabular}

Fuente: Elaboración propia.

Figura 4. Plantas de generación de energía eléctrica propuestas: a) CR; b) CRRD; c) CR2REG1REC; d) CR3REG2REC.

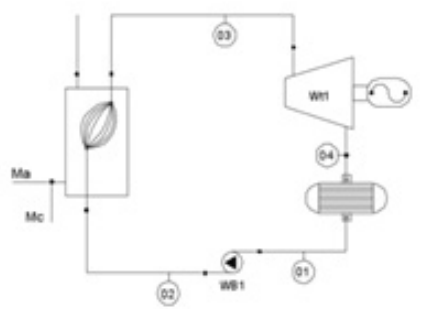

(a)

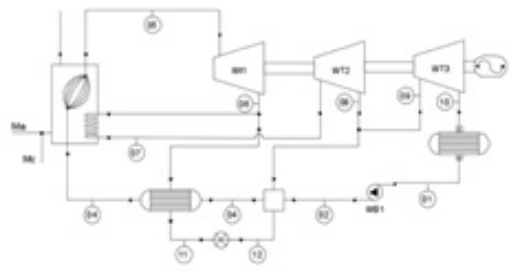

(c)

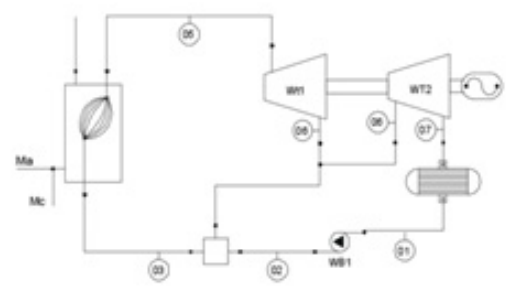

(b)

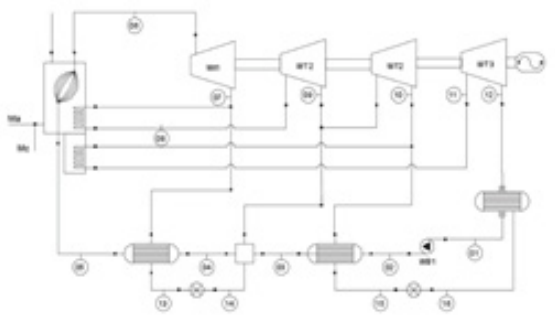

(d)

Fuente: Elaboración propia. 


\section{RESULTADOS}

En la figura 5 y tabla 2 se pueden ver los resultados del método de gravedad por resultado. Para el primer escenario, el centro de gravedad para la planta de generación se obtiene en una localización a un punto cercano de Guacalo y Olanchito, exactamente en la posición $14.9177579^{\circ}$ latitud, $-86.458191^{\circ}$ longitud. La cual tiene un costo de biomasa de US\$ 0.1098/kg.

Figura 5. Resultados de los costos ligados al centro de gravedad para el análisis energético para el escenario 1.
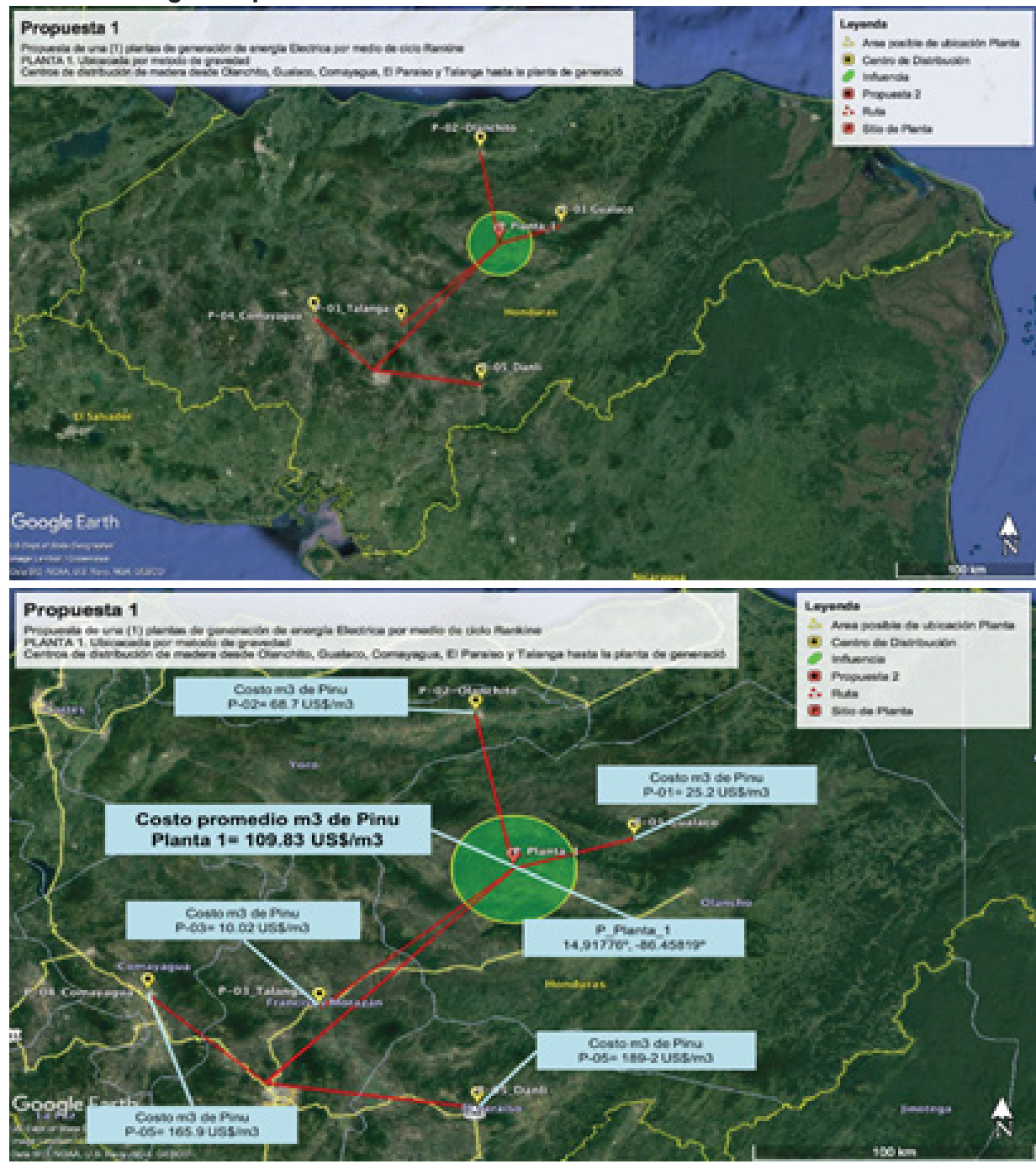

Fuente: Elaboración propia. 
Tabla 3. Resultados del centro de gravedad para el escenario 2.

\begin{tabular}{|c|c|c|c|c|c|c|c|}
\hline \multirow[t]{3}{*}{ ID } & \multirow{3}{*}{$\begin{array}{l}\text { Centro de } \\
\text { Distribución }\end{array}$} & \multicolumn{3}{|c|}{ Centro de Distribución } & \multicolumn{3}{|c|}{ Fábrica } \\
\hline & & \multicolumn{2}{|c|}{$\begin{array}{l}\text { Coordenadas del centro } \\
\text { de distribución a la Planta }\end{array}$} & \multirow{2}{*}{$\begin{array}{l}\text { Costo total } \\
\text { de la } \\
\text { Biomasa } \\
\text { (US\$ } / \mathrm{kg} \text { ) }\end{array}$} & \multicolumn{2}{|c|}{ Coordenadas de la Planta } & \multirow{2}{*}{$\begin{array}{c}\text { Costo } \\
\text { total de } \\
\text { la } \\
\text { Biomasa } \\
\text { (US\$/kg) }\end{array}$} \\
\hline & & $x$ & $\mathbf{Y}$ & & $\mathbf{x}$ & $\mathbf{Y}$ & \\
\hline \multicolumn{8}{|c|}{ Planta 1} \\
\hline 1 & Gualaco & 15.026589 & -86.071186 & 0.0252 & \multirow[t]{2}{*}{15.133186} & \multirow[t]{2}{*}{-86.192956} & \multirow[t]{2}{*}{0.0470} \\
\hline 2 & Olanchito & 15.478763 & -86.58772 & 0.0687 & & & \\
\hline \multicolumn{8}{|c|}{ Planta 2} \\
\hline 3 & Talanga & 14.402512 & -87.081734 & 0.0152 & \multirow[t]{3}{*}{14.353909} & \multirow[t]{3}{*}{-87.149344} & \multirow[t]{3}{*}{0.0690} \\
\hline 4 & Comayagua & 14.446659 & -87.63994 & 0.0843 & & & \\
\hline 5 & Danlí & 14.045031 & -86.565598 & 0.1076 & & & \\
\hline
\end{tabular}

Fuente: Elaboración propia.

En el estudio termodinámico de cada planta propuesta se presenta que independiente de la potencia neta del ciclo termodinámico sus eficiencias son completamente iguales, en la tabla A.1 de los anexos a este artículo se pueden ver esta situación. La única variación del resultado de la evaluación de estas diferentes plantas es en la mejora termodinámica implementada. En el gráfico 1, se puede ver como ocurre esta mejora dependiendo del tipo de tecnológica implementada, siendo la más eficiente la CR3REG2REC con un $35.12 \%$

Gráfico 1. Eficiencia termodinámica de las plantas de potencia propuestas

\section{Eta_term}

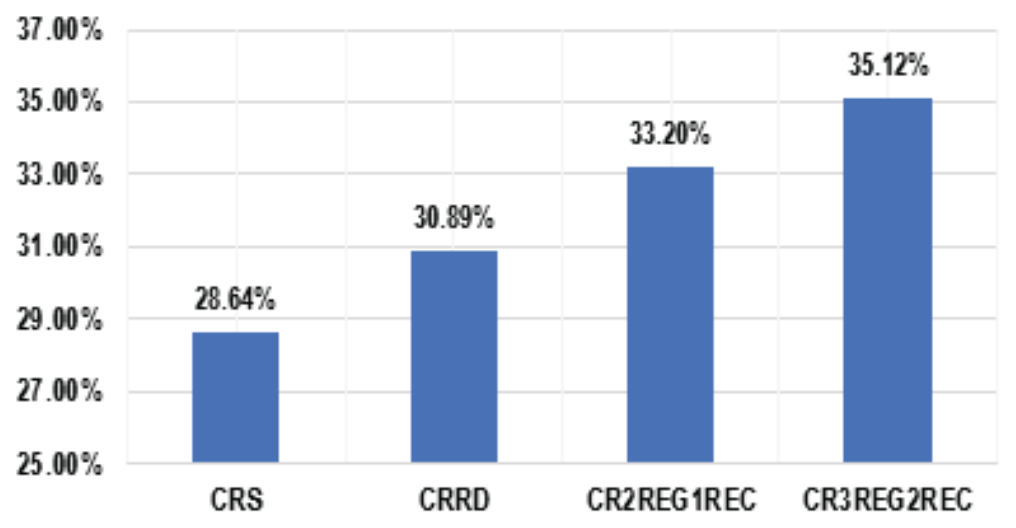

Fuente: Elaboración propia. 


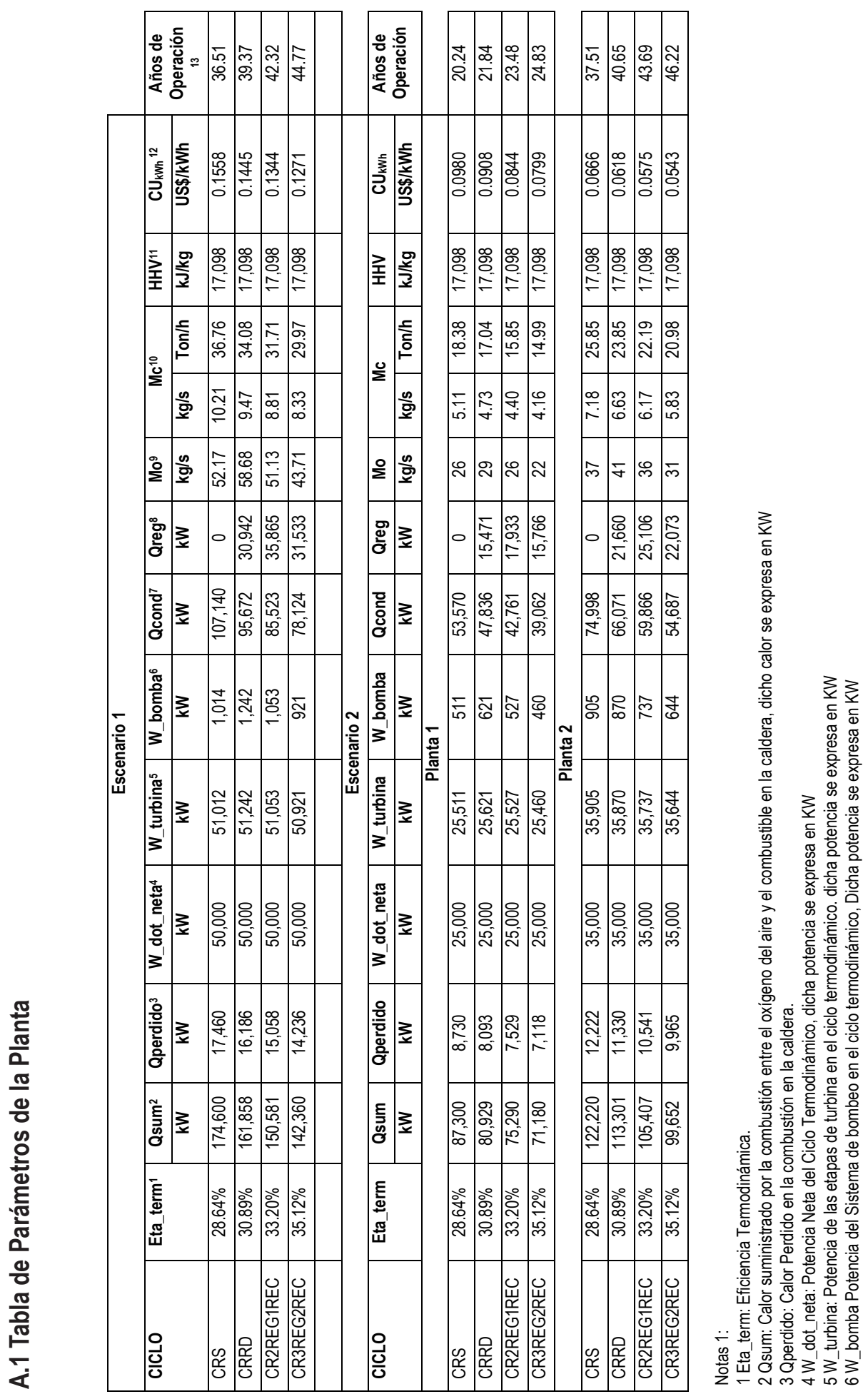


En el gráfico 2 nos muestra la reducción de combustible en Ton/h que se consume por cada planta termodinámica evaluada en el Escenario 1 y el Escenario 2, siempre siendo la CR3REG2REC con menor consumo de combustible. En el escenario 1 es necesario dosificar 29.97 ton/h para lograr una potencia neta de 50MW. Sin embargo, para el Escenario 2 donde tiene una planta de 35MW y $25 \mathrm{MW}$ donde el consumo es de 20.98 y 14.99 Ton/h respectivamente, teniendo un consumo total de $35.97 \mathrm{Ton} / \mathrm{h}$ entre las dos plantas.

\section{Gráfico 2. Consumo de combustible de las plantas de potencia propuestas}

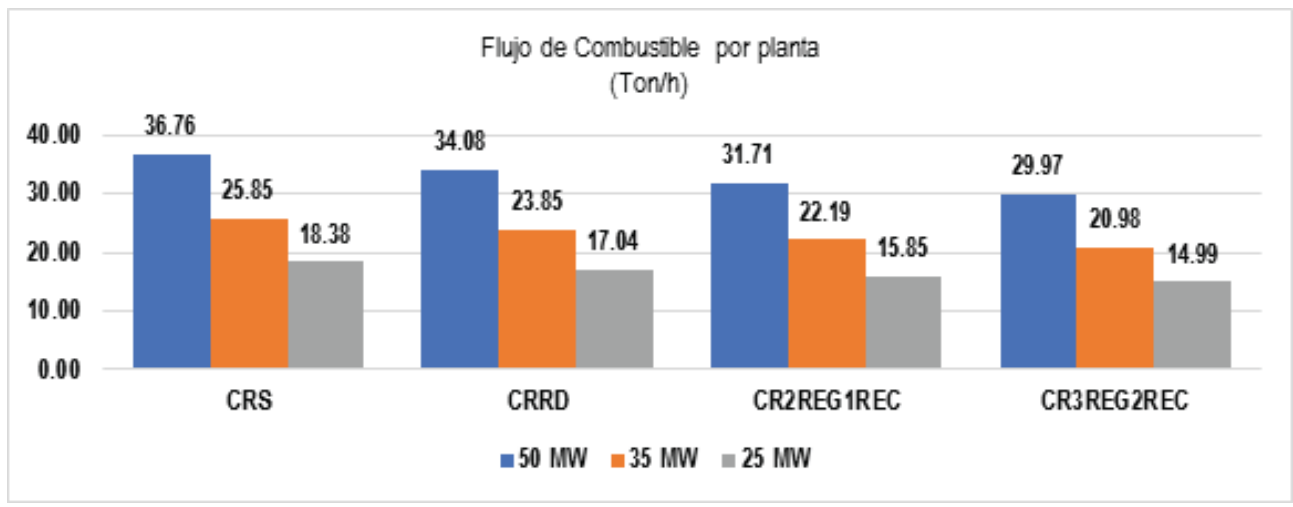

Fuente: Elaboración propia.

En el gráfico 3 nos muestra el efecto del transporte, donde la planta del escenario con mayor costo unitario del kilowatt hora ronda los US $\$ 0.1271 / \mathrm{kWh}$, siendo un valor de US $\$ 0.002542 / k w h^{*} M W$. Sin embargo, en el escenario 2 para tener un valor final de US\$0.1342/kWh para los $60 \mathrm{MW}$ totales, siendo un valor de US\$0.0022367/kWh*MW.

\section{Gráfico 3. Costo de kilowatt-hora por planta de potencia}

Costo del Kilowat-hora (US\$/kWh)

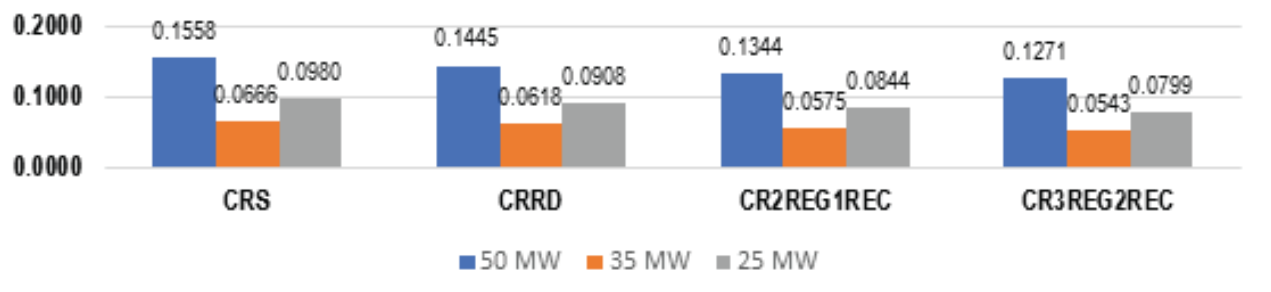

Fuente: Elaboración propia. 
Gráfico A1. Parametros termodinámicos del Escenario 1 de la planta de Potencia Neta de 50MW

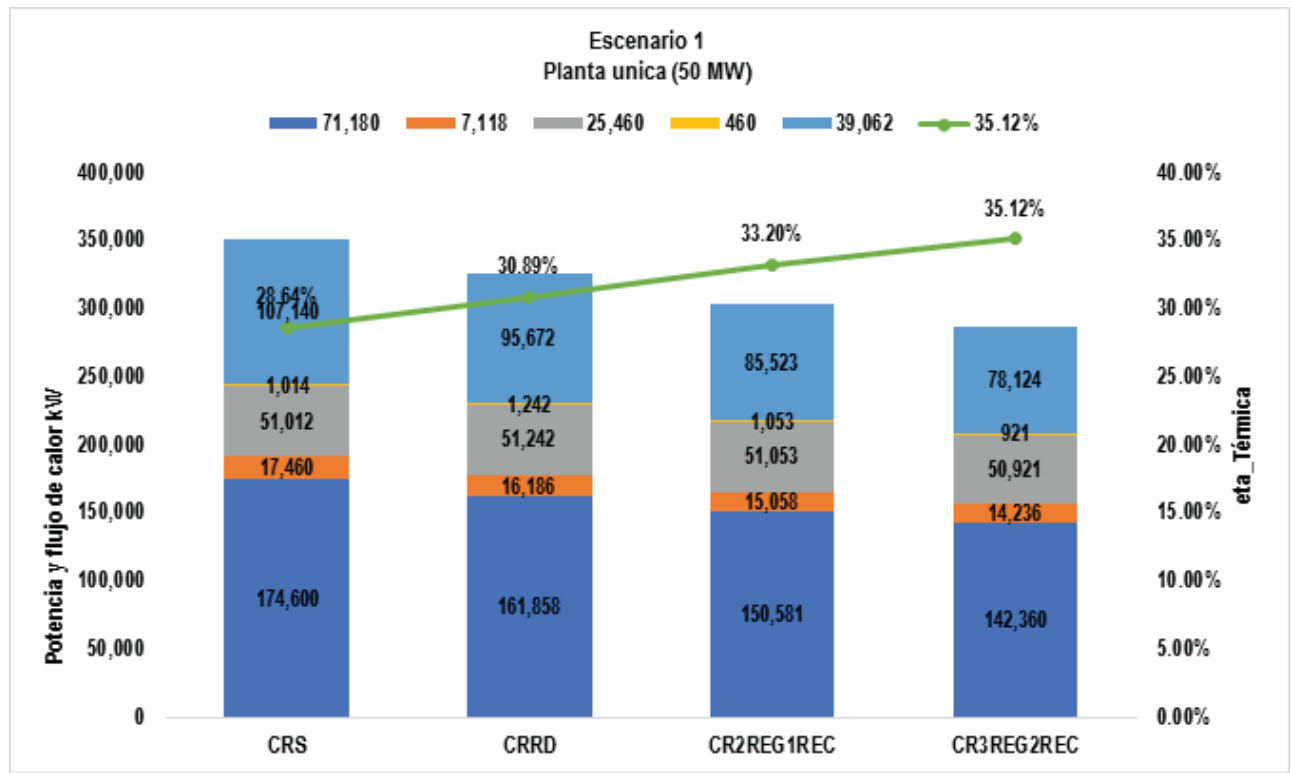

Fuente: Elaboración propia.

Gráfico A2a. Parametros termodinámicos del Escenario 2 de la planta de Potencia Neta de $35 \mathrm{MW}$

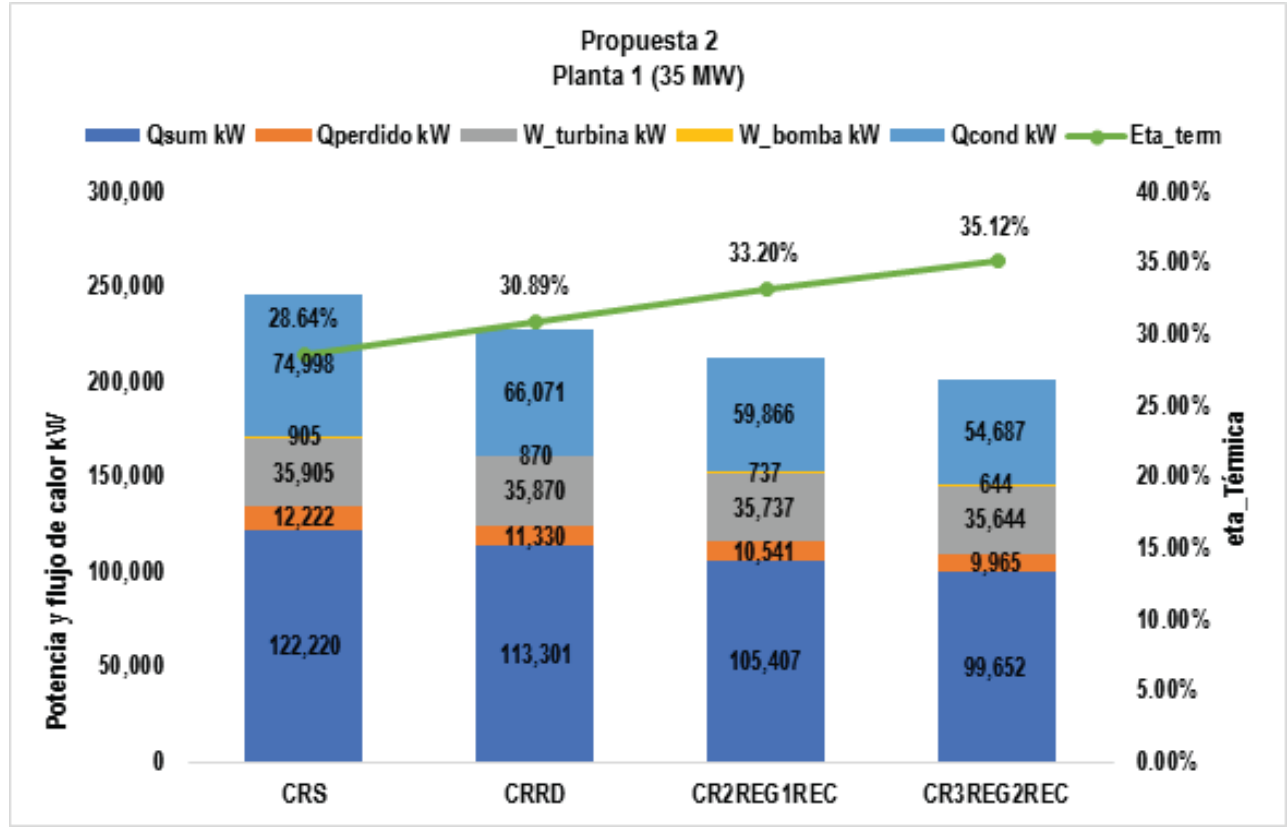

Fuente: Elaboración propia. 
Gráfico A2b. Parametros termodinámicos del Escenario 2 de la planta de Potencia Neta de $25 \mathrm{MW}$

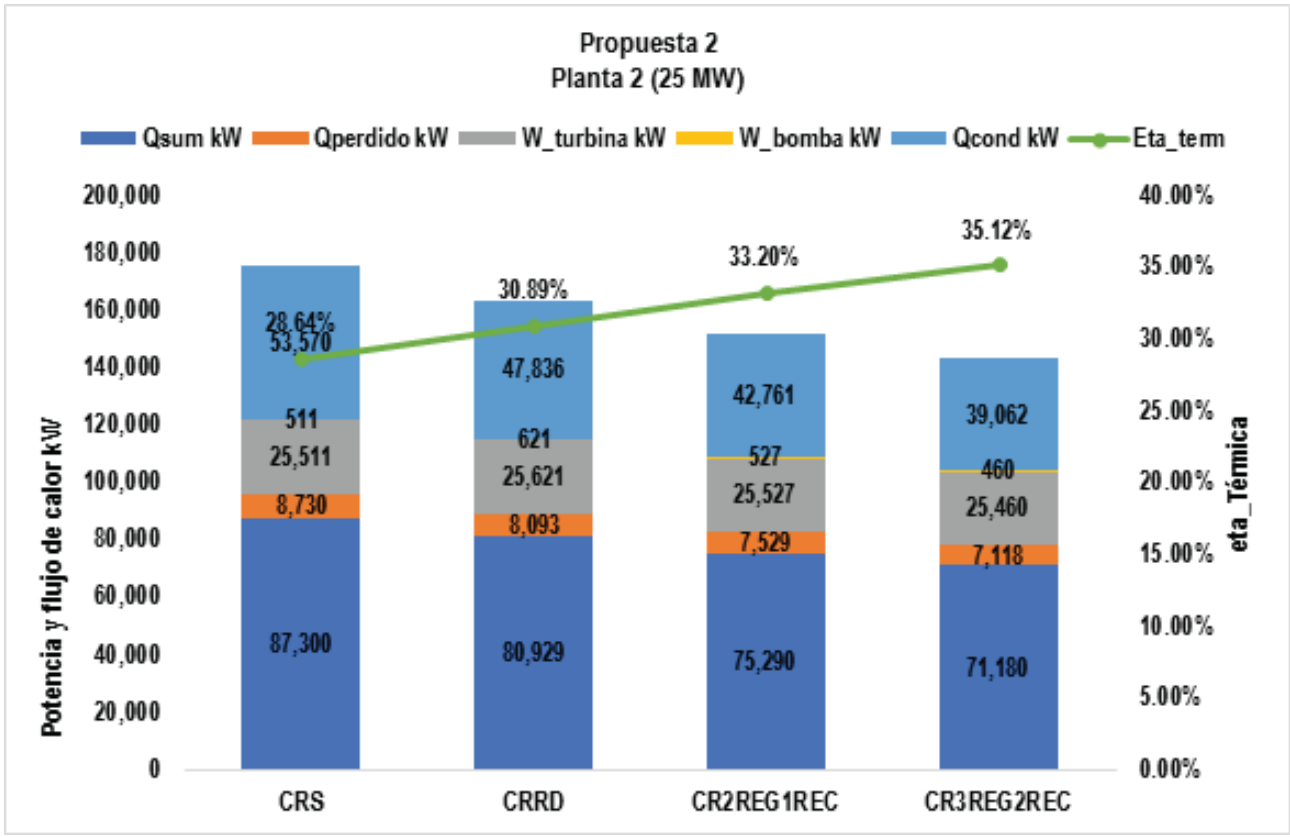

Fuente: Elaboración propia.

\section{DISCUSIÓN}

El Escenario 1 muestra que tiene un precio muy elevado de transporte, con un valor de US $\$ 0.1098 / \mathrm{kg}$, comparado con lo valores logrados por la división de potencia en el Escenario 2 con valores de costo de transporte para la planta uno y dos de US $\$ 0.04696 / \mathrm{kg}$ y US $\$ 0.0690 / \mathrm{kg}$ respectivamente.

El Ciclo termodinámico CR3REG2REC es el que muestra mejor rendimiento térmico, logrando reducir al mínimo los valores de consumo de combustible, lo cual nos muestra que con un $32.12 \%$ se obtienen la misma eficiencia independiente de la potencia neta divida entre los diferentes escenarios.

En el caso para el Escenario 1 del escenario el valor de CUkwh por MW es de US\$ 0.002542/kwh*MW, lo tanto por cada $10 \mathrm{MW}$ la potencia del escenario 1 generado con un ciclo tipo CR3REG2REC sería 0.02542/kwh. Si la producción final es de $60 \mathrm{MW}$ el costo de kWh sería de \$US 0.15252kWh. En cambio, para el Escenario 2, 
se muestra que la planta 1 de 35MW tiene un costo de producción de US\$ $0.00226 / \mathrm{kWh}^{*} \mathrm{MW}$ y para la planta 2 de $25 \mathrm{MW}$ tiene un costo de producción de US $0.002172 / \mathrm{kWh}{ }^{*} \mathrm{MW}$, el costo del kWh para ambas plantas es de US\$0.079/kWh y US\$0.0543 respectivamente.

Si lo comparamos con otras plantas térmicas mucho más eficientes como lo indica Agazzani \& Massardo (1997) que son plantas de potencia del tipo combinado Brayton/Rankine, las cuales tienen una eficiencia termica que rondan los $50 \%$, logran una reducción significativa en el costo de producción del kWh. En el estudio muestra que la generación propuesta para el análisis de ellos son tres tipos de plantas, las cuales difieren entre si en la configuración de las etapas de presión en la turbina de vapor, las cuales son: a) Un nivel de presión (LP); b) dos niveles de presión con recalentamiento (2LP-R); y c) tres niveles de presión con recalentamiento (3LP-R). El costo totales de producción de energía electrica para ellos ronda los $\$ 0.03426 / \mathrm{kWh}-\$ 0.03496 / \mathrm{kWh}$, los cuales son valores bajos por la alta eficiencia que maneja el ciclo termodinamico que propone el autor. Es importante, apuntar que el costo de inversión para este tipo de ciclos combinados son muy elevados.

Sin Embargo, se analizó la propuesta de este articulo con otros posibles sistema de generación; como un sistema de trigeneración de energía por medio de biomasa planteado por Lian, Chua, \& Chou (2010). En este caso, el mejor rendimiento en el costo de producción de energía electrica para ellos, fue el caso 3 con US $\$ 0.01524 / \mathrm{kWh}^{*} \mathrm{MW}$, muy por encima del costo que se genera plantea los escenarios en este articulo.

\section{CONCLUSIONES}

La mejor ubicación para la localización de las plantas de potencia que consumen biomasa como fuente energética se logra centralizando las plantas un lugar donde se tenga la mayor cantidad de biomasa disponible y cerca para instalar la planta. Entre más lejos es la ubicación de la planta, mayor será el CUkWh. Así mismo, es importante estar claro que la reducción del CUkWh esté ligado al ciclo termodinámico de la planta de potencia, es importante definir un ciclo que pueda ser competitivo a los valores de venta de otras fuentes energéticas, puesto que con un Ciclo termodinámiCo CR3REG2REC para 60MW zonificados en $35 \mathrm{MW}$ y $25 \mathrm{MW}$ en US\$ 0.0543/kWh y 0.0799 US\$/kWH respectivamente. 
Por tal motivo sería el Escenario 2 el que mejor se adapta a la solución del problema de consumir madera afectada para dendroctunus frontalis.

\section{REFERENCIAS BIBLIOGRAFÍCAS}

Ballou, R. H. (1973). Potencial Error in the Center Gravity in the Approach Location Facility. Transportation Journal, 13(2), 44-50.

Çengel, Y. A. (2012). Termodinámica. McGraw-Hill Interamericana de España S.L. Retrieved from https://books.google.hn/books?id=z6FbLwEACAAJ

ICF. (2017). Anuario Estadístico Forestal de Honduras 2016. Tegucigalpa. Retrieved from http://icf.gob.hn/wp-content/uploads/2017/11/Anuario-Estadistico-Forestal-2016.pdf

Kaushik, S. C., Reddy, V. S., \& Tyagi, S. K. (2011). Energy and exergy analyses of thermal power plants: A review. Renewable and Sustainable Energy Reviews, 15(4), 1857-1872. https://doi.org/10.1016/j.rser.2010.12.007

Lian, Z. T., Chua, K. J., \& Chou, S. K. (2010). A thermoeconomic analysis of biomass energy for trigeneration. Applied Energy, 87(1), 84-95. https://doi.org/10.1016/j.apenergy.2009.07.003

Oxford Dictionary. (2018). centre-of-gravity method. Retrieved from http://www.oxfordreference.com/view/10.1093/oi/authority.20110803095558367

Parikh, J., Channiwala, S. A., \& Ghosal, G. K. (2005). A correlation for calculating HHV from proximate analysis of solid fuels. Fuel, 84(5), 487-494. https://doi.org/10.1016/j.fuel.2004.10.010

Rafael Barreda del Campo, E., Augusto Araújo da Gama Cerqueira, S., \& Nebra, S. A. (1998). Thermoeconomic analysis of a Cuban sugar cane mill. Energy Conversion and Management, 39(16), 1773-1780. https://doi.org/10.1016/S0196-8904(98)00080-6

Saidur, R., Abdelaziz, E. A., Demirbas, A., Hossain, M. S., \& Mekhilef, S. (2011). A review on biomass as a fuel for boilers. Renewable and Sustainable Energy Reviews, 15(5), 2262-2289. https://doi.org/10.1016/j.rser.2011.02.015

Taner, T., \& Sivrioglu, M. (2017). A techno-economic \& cost analysis of a turbine power plant: A case study for sugar plant. Renewable and Sustainable Energy Reviews, 78(May), 722-730. https://doi.org/10.1016/j.rser.2017.04.104

Thatcher, R. C., \& Connor, M. D. (1985). Identification and biology of Sourthern Pine Bark Bertles囚: Washignton. Retrieved from https://naldc.nal.usda.gov/download/CAT89231728/PDF

Uysal, C., Kurt, H., \& Kwak, H. Y. (2017). Exergetic and thermoeconomic analyses of a coal-fired power plant. International Journal of Thermal Sciences, 117, 106-120. https://doi.org/10.1016/j.ijthermalsci.2017.03.010

Whalley, S., Klein, S. J. W., \& Benjamin, J. (2017). Economic analysis of woody biomass supply chain in Maine. Biomass and Bioenergy, 96, 38-49. https://doi.org/10.1016/j.biombioe.2016.10.015 